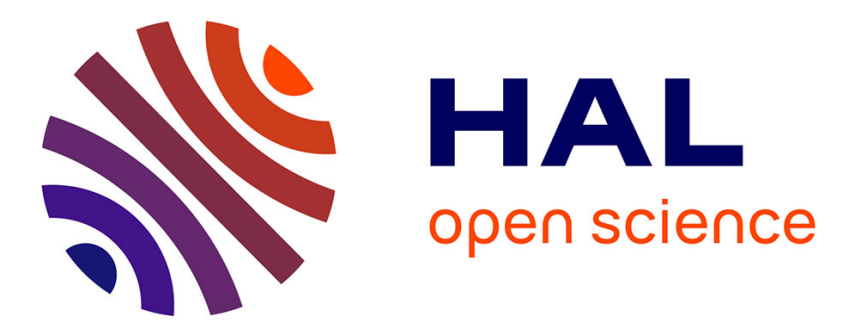

\title{
Optimization of the pumping laser beam spatial profile in the MEOP experiment performed at elevated $\mathrm{He}$ pressures
}

T. Dohnalik, A. Nikiel, T. Palasz, M. Suchanek, G. Collier, M. Greńczuk, B. Glowacz, Z. Olejniczak

\section{To cite this version:}

T. Dohnalik, A. Nikiel, T. Palasz, M. Suchanek, G. Collier, et al.. Optimization of the pumping laser beam spatial profile in the MEOP experiment performed at elevated He pressures. European Physical Journal: Applied Physics, 2011, 54 (2), 10.1051/epjap/2011100493 . hal-00698842

\section{HAL Id: hal-00698842 https://hal.science/hal-00698842}

Submitted on 18 May 2012

HAL is a multi-disciplinary open access archive for the deposit and dissemination of scientific research documents, whether they are published or not. The documents may come from teaching and research institutions in France or abroad, or from public or private research centers.
L'archive ouverte pluridisciplinaire HAL, est destinée au dépôt et à la diffusion de documents scientifiques de niveau recherche, publiés ou non, émanant des établissements d'enseignement et de recherche français ou étrangers, des laboratoires publics ou privés. 


\title{
Optimization of the pumping laser beam spatial profile in the MEOP experiment performed at elevated ${ }^{3} \mathrm{He}$ pressures
}

\author{
T. Dohnalik ${ }^{1 a}$, A. Nikiel ${ }^{1}$, T. Pałasz ${ }^{1}$, M. Suchanek ${ }^{2}$, G. Collier ${ }^{1}$, M. Greńczuk ${ }^{1}$, B. Głowacz ${ }^{1,3}$, and Z. Olejniczak ${ }^{1,4}$ \\ 1 M. Smoluchowski Institute of Physics, Jagiellonian University, Reymonta 4, 30-059 Kraków, Poland \\ 2 Physics Department, Agricultural University, Al. Mickiewicza 21, 31-120 Kraków, Poland \\ 3 Laboratoire Kastler Brossel, École Normale Supérieure, 24 rue Lhomond, 75005 Paris, France \\ 4 Institute of Nuclear Physics, Polish Academy of Sciences, Radzikowskiego 152, 31-342 Kraków, Poland
}

Received: date / Revised version: date

\begin{abstract}
The efficiency of the metastability exchange optical pumping (MEOP) performed at elevated ${ }^{3} \mathrm{He}$ gas pressures in high magnetic field was improved by modifying the spatial profile of the pumping laser beam in such a way that it matched the nonuniform distribution of plasma in the optical pumping cell. Comparing to a conventional Gaussian profile, at the ${ }^{3} \mathrm{He}$ gas pressure equal to $267 \mathrm{mbar}$, both the achieved nuclear polarization (equal to $26 \%$ ) and the total magnetization in the cell (equal to 1.37 sccfp) are $60 \%$ higher when the annular laser beam profile is applied.
\end{abstract}

PACS. XX.XX.XX No PACS code given

\section{Introduction}

For many years, nuclearly polarized ${ }^{3} \mathrm{He}$ gas has been used in fundamental research for generation and analysis of polarized neutrons in nuclear physics [1-3]. Recently, a new and exciting application for hyperpolarized ${ }^{3} \mathrm{He}$ gas has been found in magnetic resonance imaging (MRI) of human lungs [4-7]. This new application motivated the studies aiming at the optimization of the ${ }^{3} \mathrm{He}$ polarization techniques, in order to produce large amounts of highly polarized ${ }^{3}$ He gas needed in medical applications. Two methods can be used for this purpose: spin exchange optical pumping (SEOP) [8], and metastability exchange optical pumping (MEOP) [9], which is the subject of this paper. In the MEOP process, the ${ }^{3} \mathrm{He}$ atoms in the ground state are excited into the metastable $2^{3} \mathrm{~S}$ state by a weak rf discharge. The optical pumping of the metastable atoms is performed by circularly polarized light at $1083 \mathrm{~nm}$ wavelength, corresponding to the $2^{3} \mathrm{~S}-2^{3} \mathrm{P}$ transition. The nuclear polarization of the ground state ${ }^{3} \mathrm{He}$ atoms is achieved by metastability exchange collisions with optically pumped ${ }^{3} \mathrm{He}$ atoms in the $2^{3} \mathrm{~S}$ metastable state.

A low magnetic field ( $1 \mathrm{mT}$ ) and low ${ }^{3} \mathrm{He}$ gas pressure ( 1 mbar) are the standard conditions for the MEOP process. At higher pressure, the two-body ionizing collisions and three-body collisions producing metastable helium molecules dramatically decrease the population of metastable atoms, reducing the efficiency of the MEOP process $[10,11]$. In the MRI of human lungs, the gas to be inhaled should be at atmospheric pressure, therefore

\footnotetext{
a e-mail: tomasz.dohnalik@uj.edu.pl
}

the hyperpolarized ${ }^{3} \mathrm{He}$ gas needs to be first compressed to about 100 mbar, and then mixed with another buffer gas, like nitrogen and/or ${ }^{4} \mathrm{He}$, which leads to a ten-fold decrease of the final magnetization density. It would be beneficial to produce the polarized ${ }^{3} \mathrm{He}$ gas directly at higher pressure, say about 100 mbar. That would also relax the demanding technical requirements for the initial compression, both in terms of compression time and polarization losses. Recently, it has been shown that the MEOP method can be used at elevated ${ }^{3} \mathrm{He}$ gas pressure, provided it is performed at high magnetic field [10-15]. Qualitatively, this effect can be explained by quenching of the hyperfine interaction in the presence of high magnetic field. In standard conditions of low magnetic field, the hyperfine coupling causes some nuclear polarization losses by back transfers of nuclear orientation to electronic orientations. The high magnetic field decoupling minimizes this effect, counterbalancing the reduction of population of metastable atoms at higher pressure caused by collisions. However, the processes are not fully understood and are still the subject of active research $[14,15]$. Until now, the highest pressures for which MEOP has been successfully employed are 64 mbar at $2 \mathrm{~T}$ and $266 \mathrm{mbar}$ at $4.7 \mathrm{~T}$, and the polarizations reported were $52 \%$ and $22 \%$, respectively $[15,16]$. These promising results motivated us to pursue the high-field experimental studies even further, the final goal being the design of a high-field MEOP polarizer that would produce large quantities of highly polarized ${ }^{3} \mathrm{He}$ gas directly in the magnetic field of the MRI medical scanner.

In this paper we show that a further improvement of MEOP performance at high magnetic field is feasible, by 


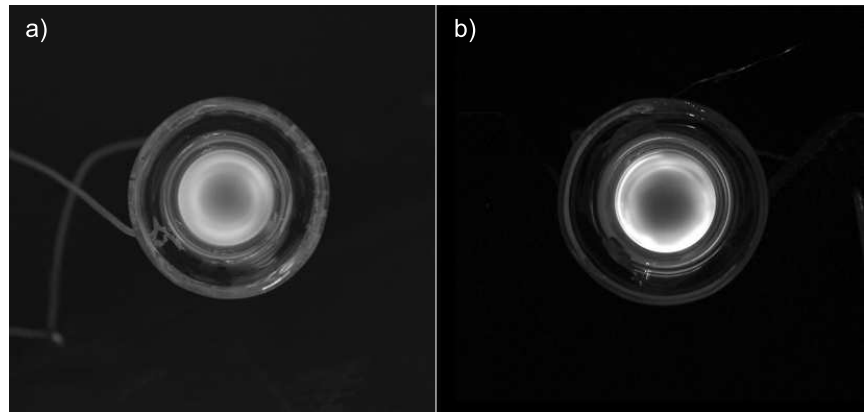

Fig. 1. Photographs of optical pumping cells loaded to $32 \mathrm{mbar}$ (a) and 128 mbar (b) of ${ }^{3} \mathrm{He}$ gas with the discharge turned on.

taking into account a nonuniform distribution of plasma in the optical pumping cell that is observed at elevated pressures and/or high magnetic field. The pumping light absorption can be significantly enhanced if the beam profile matches this distribution, increasing the efficiency of the MEOP process at a given laser power. To assess the pump laser beam profile effect on the efficiency of the MEOP process, two laser sources with four optical setups were tested, using five optical pumping cells filled with the ${ }^{3} \mathrm{He}$ gas with pressure ranging from 32 to 267 mbar, in a magnetic field of $2 \mathrm{~T}$. It was found that the annular profile, which concentrates the laser power near the cell walls, gives the optimum efficiency in the whole ${ }^{3} \mathrm{He}$ gas pressure range.

\section{Radial distribution of plasma in the excited ${ }^{3} \mathrm{He}$ gas at elevated pressures in $2 \mathrm{~T}$}

When the MEOP method is performed in standard operating conditions, i.e. at low ${ }^{3} \mathrm{He}$ gas pressure and in low magnetic field, the plasma distribution is uniform in the whole optical pumping cell. Therefore a conventional Gaussian profile of the pumping laser beam is usually applied [17-19]. The situation is quite different when the optical pumping is performed at high magnetic field or at elevated pressure. In that case the plasma is generally nonuniform and is located mostly close to the walls of the cell. This effect is illustrated in Fig. 1a and 1b for the two experimental cells loaded to 32 mbar and 128 mbar, respectively. As a consequence of this effect, there is only partial overlapping of the Gaussian laser beam and population of atoms in the metastable state. A large fraction of the pumping laser power is wasted, lowering the overall efficiency of the MEOP process.

In order to analyze this effect quantitatively, systematic measurements of the resonant laser light absorption by excited ${ }^{3} \mathrm{He}$ gas were carried out over a large range of gas pressures: 32, 67, 96 and 128 mbar, in a $2 \mathrm{~T}$ magnetic field. Closed, bone-shaped, $16 \mathrm{~mm}$ inner diameter optical pumping cells of $110 \mathrm{~mm}$ length and with $25 \mathrm{~mm}$ diameter windows were used. A $2 \mathrm{MHz}$ radio frequency signal was applied to two electrodes that were wound on the outer surface of the cells to generate the discharge in the gas. Depending on rf amplitude, the metastable

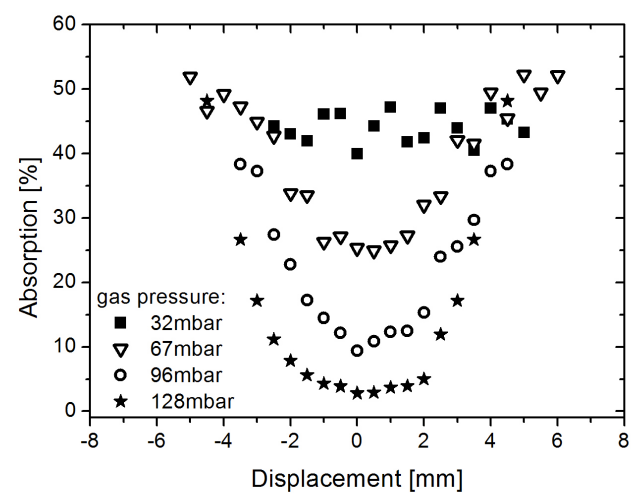

Fig. 2. Radial dependence of the resonant laser absorption for various ${ }^{3} \mathrm{He}$ gas pressures at $2 \mathrm{~T}$.

atom density was in the $10^{10}-10^{12}$ atoms $/ \mathrm{cm}^{3}$ range. $\mathrm{A}$ Distributed Bragg Refraction (DBR) laser was employed, generating a Gaussian beam with FWHM of $10.2 \mathrm{~mm}$. A nonmagnetic 3D displacement system with the attached photodiode was located inside the magnet, $2 \mathrm{~cm}$ behind the optical pumping cell. The absorbed energy along the horizontal radial axis of the cell was determined, in 0.5 mm steps. For each data point three measurements of the light intensity were made. The absorption was then calculated from the following expression:

$$
\text { Absorption }=\frac{I_{1}-I_{2}}{I_{1}-I_{0}},
$$

where $I_{0}$ is the signal from photodiode when both the laser and discharge are off (noise signal), $I_{1}$ when the laser is on and discharge off (background signal), and $I_{2}$ when both laser and discharge are on (the presence of metastable atoms). In that way the calculated absorption is independent of the laser beam profile.

The resulting resonant laser absorption profiles measured at various ${ }^{3} \mathrm{He}$ gas pressures are shown in Fig. 2. Due to some undesired effects, like reflections and scattering of the laser light, the measurements close to the cell walls were unreliable and are not shown. For the lowest gas pressure of 32 mbar the radial distribution of the plasma is nearly uniform, but it exhibits a minimum at the center of the cell, which deepens with increasing pressure. The laser light absorption level along the longitudinal axis of the cell approaches zero at 128 mbar. On the other hand, it stays high close to the walls of the cell and is quite independent of the gas pressure. The absorption profiles reflect the distribution of metastable atoms in the plasma, which in turn are responsible for the optical pumping. Therefore, in order to optimize the efficiency of the MEOP process, the spatial shape of the pumping laser beam should match the plasma distribution. This was the main motivation for undertaking systematic studies of the pumping beam profile effects as a function of ${ }^{3} \mathrm{He}$ gas pressure, discharge intensity and laser power in the magnetic field of $2 \mathrm{~T}$. 
a)

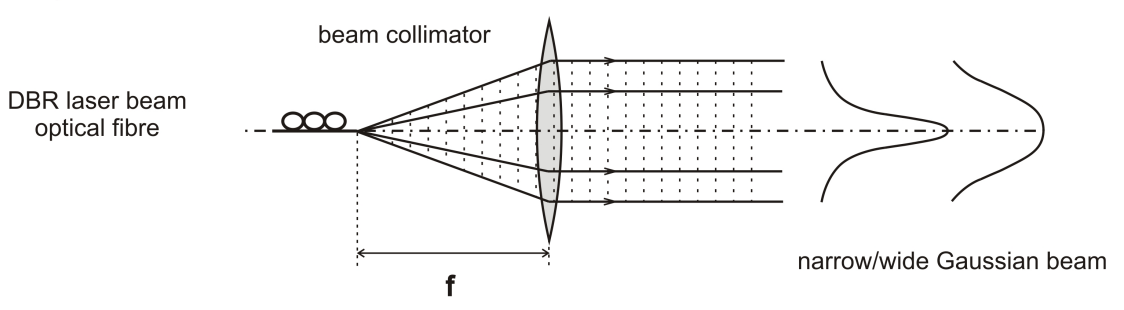

b)

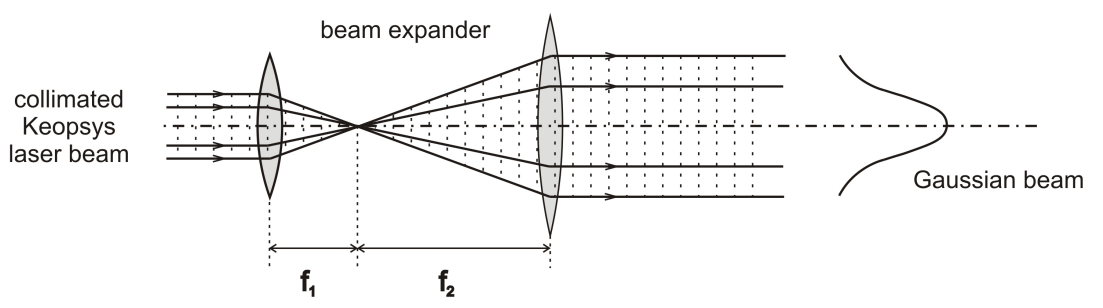

c)

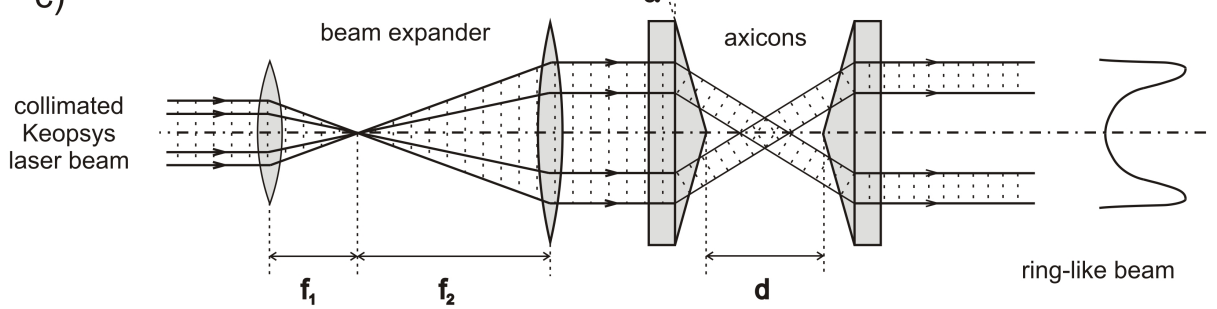

Fig. 3. Schematic arrangement for the pump laser beam forming: (a) Gaussian profile by using a collimator, (b) expanded Gaussian profile by using the Kepler-like telescope, (c) annular profile by using a pair of axicons.

Table 1. Specifications of optical setups used for pump laser beam forming (for symbol description see Fig. 3).

\begin{tabular}{lllll}
\hline laser source & expander used & $\begin{array}{l}\text { optical setup } \\
\text { parameters }\end{array}$ & $\begin{array}{l}\text { FWHM } \\
{[\mathrm{mm}]}\end{array}$ & $\begin{array}{l}\text { intensity } \\
\text { distribution }\end{array}$ \\
\hline amplified DBR diode laser & collimator & $\mathrm{f}=30 \mathrm{~mm}$ & 3.2 & Gaussian \\
amplified DBR diode laser & collimator & $\mathrm{f}=100 \mathrm{~mm}$ & 10.2 & $\begin{array}{l}\text { Gaussian } \\
\text { Keopsys }\end{array}$ \\
Keopsys & axicon-based expander & $\mathrm{f} 2 / \mathrm{f} 1=7$ & 4.9 & Gaussian \\
& & $\mathrm{d}=205 \mathrm{~mm} ; \alpha=10^{0}$ & & annular \\
\hline
\end{tabular}

\section{Generation of various pump laser beam spatial profiles}

The optical setups used in the present studies are illustrated in Fig. 3 and their parameters are summarized in Table 1. Two laser sources were used in our experiments for optical pumping. In the first stage, a comparison of the narrow and wide Gaussian beam profiles was made using a $50 \mathrm{~mW}$ single mode DBR laser diode (SDL6702-H1), followed by the ytterbium doped fiber amplifier (IPG, Burbach, Germany), which produced up to 0.5 $\mathrm{W}$ of tunable laser light [20]. To expand the Gaussian beam from the DBR diode laser, a collimating lens was applied, which was located at the distance equal to the focal length from the output of the laser fiber (Fig. 3a). Parallel beams characterized by Gaussian radial profiles with the FWHM of about $3.2 \mathrm{~mm}$ (narrow beam) and $10.2 \mathrm{~mm}$ (wide beam) were generated, using lenses with focal lengths equal to $30 \mathrm{~mm}$ and $100 \mathrm{~mm}$, respectively. In the second stage, a new ytterbium doped fiber laser (Keopsys CUS-BT-YFL-1083-HE-100-COL, France) with the wavelength of $1083 \mathrm{~nm}$ was employed, producing up to $10 \mathrm{~W}$. It was used to compare the performance of the Gaussian and annular pumping laser beam profiles. The expansion of $10 \mathrm{~W}$ Keopsys laser beam was made either by a Kepler-type telescope that consisted of two conver- 
gent lenses both at the input and output of the expander (Fig. 3b), or by the same telescope followed by a pair of convergent axicons (Fig. 3c). In the first case the telescope with magnification of about 7 was used, resulting in a Gaussian intensity profile with the FWHM of about $4.9 \mathrm{~mm}$.

The annular profile of the laser beam that should match the distribution of metastable atoms in the optical pumping cell was created by a pair of axicons (Fig. 3c). The axicon term was suggested for the first time by J.H. McLeod, to define refractive or diffractive cylindrically symmetric optical elements [21]. Nowadays the most popular axicon is in the form of a conical prism. A pair of axicons can transform the Gaussian beam into an annular beam without any loss of energy. The intensity distribution of the generated beam can be modified in a continuous way by simply changing the incident Gaussian beam diameter and the distance between the two prisms. The axicons used in the present work were made of BK7 glass with refractive index $\mathrm{n}=1.57$. They have one flat and one conical surface, a diameter of about $50.8 \mathrm{~mm}$, and the base angle of $10^{0}$. The tips of axicons are about $205 \mathrm{~mm}$ apart so that a ring with the outer diameter of $36 \mathrm{~mm}$ could be formed. In order to fit the size of the outgoing annular beam to the internal diameter of the optical pumping cell, a second Kepler-type telescope with magnification of $2.5^{-1}$ was employed (not shown). This resulted in the annular beam profile of outer and inner diameters equal to $14.8 \mathrm{~mm}$ and $10 \mathrm{~mm}$, respectively.

The actual laser beam intensity profiles for all optical arrangements were measured, using a similar setup as in Section 2, but outside of the magnet, and directly on the attenuated laser beam. The photodiode was moved along the transverse axis at $0.5 \mathrm{~mm}$ steps, and the signal was measured by a digital voltmeter. The resulting radial intensity distributions for the DBR diode laser and for the Keopsys laser are shown in Fig. 4a and b, respectively. In both Figures, the vertical lines represent the transverse dimension of the optical pumping cell. For the first three cases, Gaussian fits to experimental points were made (solid lines), and the resulting widths are reported in Table 1. The annular profile generated by the axicons exhibits some oscillations, which can be caused by diffraction effects occurring at the sharp edges of axicons, imperfect axicon tips [22], as well as a small misalignment of optical elements.

\section{MEOP experiments}

Extensive MEOP measurements were carried out using a $2 \mathrm{~T}$ superconducting magnet (Magnex Scientific) and implementing various versions of the pumping laser beam profile. The experimental arrangement is depicted in Fig. 5 and is similar to that used in the previous study [15]. The place of the optical setup modification is shown schematically in the Figure. The pumping cell and the optical apparatus were mounted on a non-magnetic table inside the $30 \mathrm{~cm}$ diameter bore of the magnet. The lasers and the electronic equipment were located a few meters away in a a)

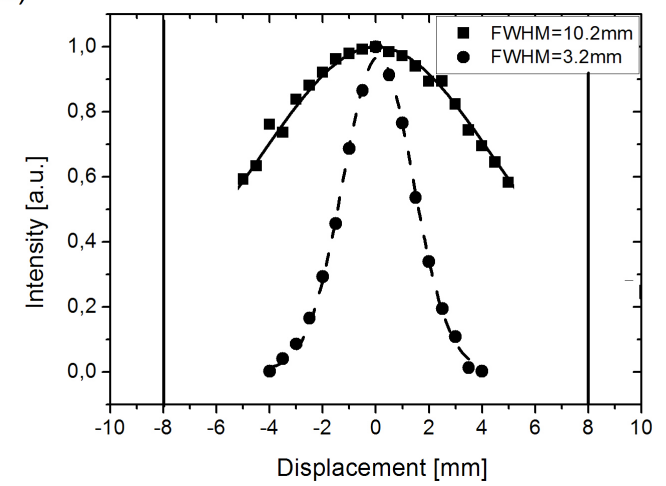

b)

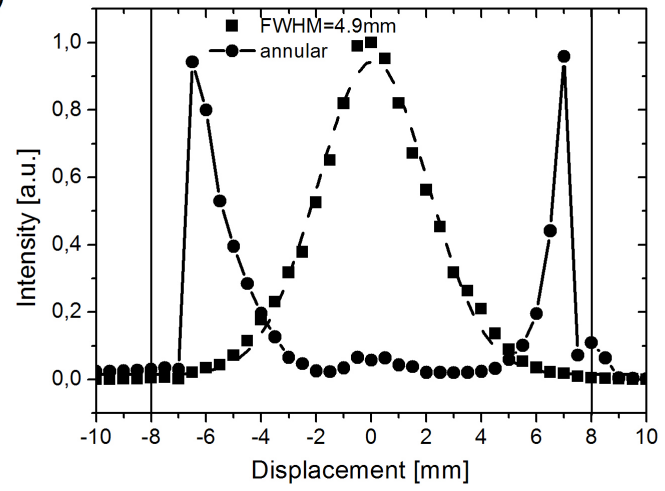

Fig. 4. Transverse intensity profiles (a) of the narrow and wide Gaussian generated by the DBR diode laser, (b) of the Gaussian and ring-like beam generated by the Keopsys laser.

low magnetic field region. The bone-shaped optical pumping cells filled with ${ }^{3} \mathrm{He}$ under 32, 64, 96, 128 and 267 mbar pressure were used. A weak rf discharge populated the $2^{3} \mathrm{~S}$ metastable state. For all experimental arrangements the laser wavelength was tuned to the $\mathrm{f}^{2-}$ line of $2^{3} \mathrm{~S}-2^{3} \mathrm{P}$ absorption spectrum, since it was demonstrated before that using that optical pumping transition the best MEOP results are obtained [15]. In each case the laser power delivered to the optical pumping cell was carefully minitored and was equal to $0.5 \mathrm{~W}$. Following the expander, the input laser beam was circularly polarized by a quarter wave plate and directed towards the optical pumping cell, then back-reflected by a mirror to enhance its absorption by the ${ }^{3} \mathrm{He}$ gas, and monitored by a photodiode. In order to eliminate the long tails of the Gaussian beam profiles, an additional diaphragm with $11 \mathrm{~mm}$ aperture was applied in front of the optical pumping cell.

In order to measure the nuclear polarization $M$ of ${ }^{3} \mathrm{He}$ atoms, a second DBR diode laser was implemented to produce a weak $\left(\sim 100 \mu \mathrm{W} / \mathrm{cm}^{2}\right)$ probe beam of $1.5 \mathrm{~mm}$ diameter. The circularly polarized probe beam was almost collinear with the pump beam (tilt angle $<3^{0}$ ). The discharge intensity was modulated at $120 \mathrm{~Hz}$ with $15 \%$ depth 


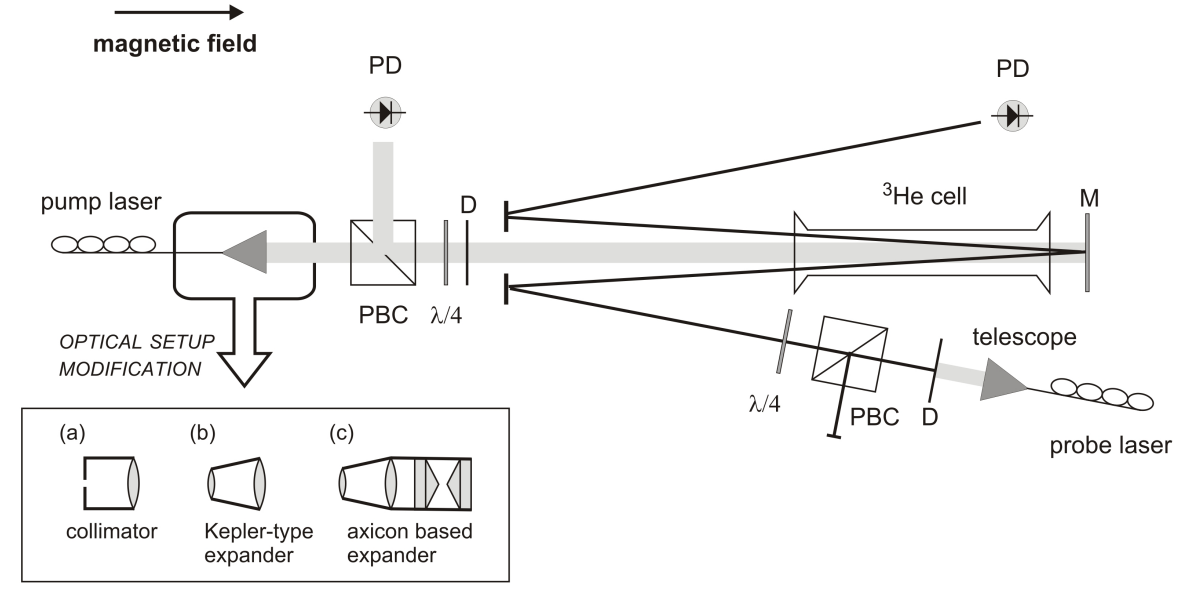

Fig. 5. The MEOP experimental setup (PBC - polarizing beam splitter cube , $\lambda / 4$ - quarter-wave plate, PD - photodiode, $\mathrm{M}$ - mirror, D - diaphragm). The three alternative optical beam formers are indicated.

to measure the probe beam absorption with the lock-in amplifier. The optical method to measure $M$ is described in detail in [23].

The numerical results of all experiments are summarized in Table 2 . In the Figure $6 \mathrm{a}(6 \mathrm{~b})$ the relation between the steady-state nuclear polarization $M$ (the total magnetization $M_{t o t}$ in standard cubic centimeters of fully polarized gas, sccfp) and the gas pressure for four different intensity profiles of the pump laser beam is illustrated. For all cases, the steady-state nuclear polarization decreases with increasing ${ }^{3} \mathrm{He}$ gas pressure, but it is systematically higher when the ring-like beam generated by the new $10 \mathrm{~W}$ Keopsys laser is applied. The highest value of total magnetization (which is proportional to the NMR signal) obtained during the experiments was equal to 1.37 sccfp, which is a factor of two higher than the previously reported result for the Gaussian beam [15].

The MEOP experiments for various ${ }^{3} \mathrm{He}$ gas pressures using various pumping laser beam profiles were carried out chronologically, starting from the Gaussian profiles of different widths, and finishing with the annular beam. Since it required a serious modification of experimental setup, it was difficult to reproduce the same experimental conditions for each case. Specifically, it was impossible to obtain the same plasma intensity in the cell, which would be characterized by the same relaxation time $T_{1}$. However, according to [13], in high magnetic field the steady-state polarization $M$ and total magnetization $M_{t o t}$ are independent of $T_{1}$. Therefore, for a given pressure in the cell and at fixed pumping laser power $(0.5 \mathrm{~W})$, these parameters will only depend on the pumping laser beam profile.

The MEOP performance as a function of the pumping laser power was also studied, in the $0.5-2.0 \mathrm{~W}$ range. A weak rf discharge level was used. Both the steady state nuclear polarization $M$ and the total magnetization $M_{t o t}$ show rather weak dependence on the pumping laser power. On the other hand, the build-up time decreases rapidly with the laser power, as expected. a)

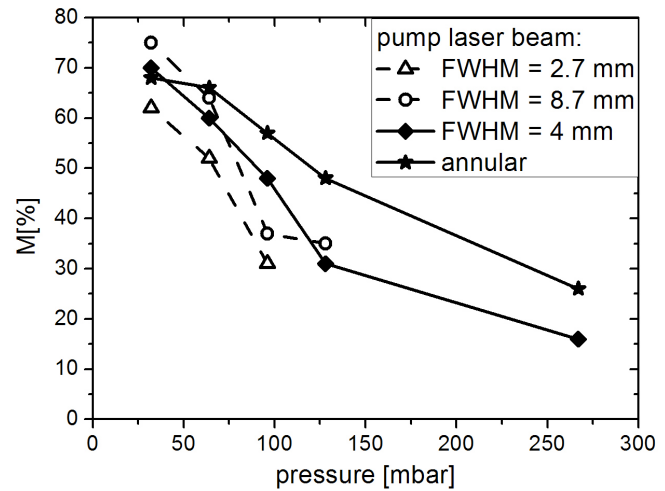

b)

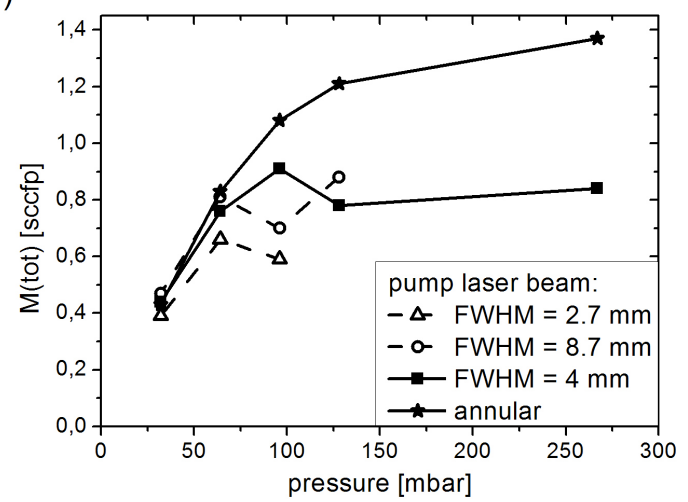

Fig. 6. Dependence of (a) the steady-state nuclear polarization $M$, and (b) the total magnetization $M_{t o t}$ on the ${ }^{3} \mathrm{He}$ gas pressure for optical pumping performed with various laser beam profiles. 
Table 2. Comparison of measured parameters: $M$ and $M_{t o t}$ for various laser beam profiles at $0.5 \mathrm{~W}$ laser power.

\begin{tabular}{|c|c|c|c|c|c|c|c|c|}
\hline \multirow[t]{3}{*}{$\begin{array}{l}\text { Pressure } \\
{[\mathrm{mbar}]}\end{array}$} & \multicolumn{4}{|c|}{$\begin{array}{l}\text { Polarization } M \\
{[\%]}\end{array}$} & \multicolumn{4}{|c|}{$\begin{array}{l}\text { Magnetization } M_{t o t} \\
{[\text { sccfp] }}\end{array}$} \\
\hline & FWHM & FWHM & FWHM & annular & FWHM & FWHM & FWHM & annular \\
\hline & $3.2 \mathrm{~mm}$ & $10.2 \mathrm{~mm}$ & $4.9 \mathrm{~mm}$ & beam & $3.2 \mathrm{~mm}$ & $10.2 \mathrm{~mm}$ & $4.9 \mathrm{~mm}$ & beam \\
\hline 32 & 62 & 75 & 70 & 68 & 0.39 & 0.47 & 0.44 & 0.43 \\
\hline 64 & 52 & 64 & 60 & 66 & 0.66 & 0.81 & 0.76 & 0.83 \\
\hline 96 & 31 & 37 & 48 & 57 & 0.59 & 0.7 & 0.91 & 1.08 \\
\hline 128 & - & 35 & 31 & 48 & - & 0,88 & 0.78 & 1.21 \\
\hline 267 & - & - & 16 & 26 & - & - & 0.84 & 1.37 \\
\hline
\end{tabular}

\section{Conclusions}

In this paper, the MEOP results obtained for five ${ }^{3} \mathrm{He}$ gas pressures $(32,64,96,128$ and 267 mbars) at the magnetic field of $2 \mathrm{~T}$ are described. This experiment continues the systematic studies of MEOP in a large range of magnetic field and pressure, in search for optimum operating conditions.

A new experimental setup consisting of the $10 \mathrm{~W}$ laser and supplemented by a pair of axicons was designed. It transforms the Gaussian beam that is routinely used in MEOP experiments into an annular beam. It optimizes the optical pumping efficiency at elevated ${ }^{3} \mathrm{He}$ gas pressures, since the intensity distribution of the pumping light is as close as possible to the metastable atom distribution in the optical pumping cell. As expected, the best performance is achieved with the ring-like beam profile, both in terms of the steady-state nuclear polarization $M$ and the total magnetization $M_{t o t}$. We have shown for the first time that with the use of this novel optical setup it is possible to polarize ${ }^{3} \mathrm{He}$ gas with high field MEOP for pressures exceeding 250 mbar while still maintaining nuclear polarization of $20 \%$ or higher. This is an important result when one considers the fact that the gas compression from a few mbars to one bar without losing too much of polarization has always been a limiting factor of the MEOP method, for its implementation on a small scale in the hospital. A central production facility of polarized ${ }^{3} \mathrm{He}$ gas was developed several years ago [24]. It uses a polarization-conserving hydraulic compressor to produce polarized ${ }^{3} \mathrm{He}$ gas up to $5-6$ bars. However, it is inconvenient for local applications due to high cost of transportation and handling.

Another reason to pump at elevated pressure in high magnetic field is that the total magnetization increases with pressure. At almost 100 mbar the nuclear polarization of $48 \%$ and the total magnetization of 0.9 sccfp was obtained. This pressure is only ten times smaller than 1 bar needed for medical applications. We believe that in a near future it will be possible to routinely polarize the ${ }^{3} \mathrm{He}$ gas in these conditions, relaxing the demanding technical requirements for the final stage compressor.

We will continue our study of MEOP performance at elevated pressures at somewhat lower magnetic field of 1.5 T. A longer pumping cell will be tested at higher laser power. This will enlarge the experimental basis for a rigor- ous test of the theoretical model of MEOP process in high magnetic field. Hopefully, this will also lead to the determination of optimum operating conditions for a portable high-field polarizer that could work in the magnetic field provided by the MRI medical scanner. A prototype of such a device is under construction in our laboratory.

\section{Acknowledgements}

The optical pumping cells that were used in this work were lent to us by Pierre-Jean Nacher and Genevieve Tastevin from LKB in Paris. We greatfully acknowledge their continuous support for our project. This work was partially supported by the Polish Ministry of Science and Higher Education (Grant MRTN-CT-2006-036002). GC is the MarieCurie fellow in the PHeLINet network. AN was supported by the graduate student fellowship from the Polish Ministry of Science and Higher Education (Grant NN 202006734).

\section{References}

1. P. L. Anthony, Phys.Rev. Lett. 71, (1993) 959.

2. N. R. Newbury, A. S. Barton, P. Bogorad, G. D. Cates, M. Gatzke, B. Saam, L. Han, R. Holmes, P. A. Souder, J. Xu, D. Benton, Phys.Rev. Lett. 67, (1991) 3219.

3. G.L. Jones, T. Gentile, A.K. Thompson, Z. Chowdhuri, M.S. Dewey, W.M. Snow, F.E.Wietfeldt, Nucl. Instrum. Meth. A 440, (2000) 772.

4. P. Bachert, L. R. Schad, M. Bock, M. V. Knop, M. Ebert, T. Grossmann, W. Heil, D. Hofmann, R. Surkau and E. W. Otten, Magn. Reson. Med. 36, (1996) 192.

5. M. Ebert, T. Grossmann, W. Heil, E. Otten, R. Sarkau, M. Leduc, P. Bachert, M. Knopp, L. Schad, M. Thelen, Lancet 34, (1996) 1297.

6. H.E. Möller, X.J. Chen, B. Saam, K.D. Hagspiel, G.A. Johnson, T.A. Altes, E.E. de Lange, H.U. Kauczor, Magn. Res. Med. 47, (2002) 1029.

7. H.-U. Kauczor, MRI of the lung (Springer-Verlag Berlin and Heidelberg GmbH \& Co. KG 2009)

8. T. G. Walker, W. Happer, Rev. Mod. Phys. 69, (1997) 629.

9. F. D. Colegrove, L. D. Schearer, G. K. Walters, Phys. Rev. 132, (1963) 2561.

10. P.-J. Nacher, E. Courtade, M. Abboud, A. Sinatra, G. Tastevin, T. Dohnalik, Acta Phys. Pol. B 3, (2002) 2225. 
11. E. Courtade, F. Marion, P. J. Nacher, G. Tastevin, K. Kiersnowski, T. Dohnalik, Eur. Phys. J. D 21, (2002) 25.

12. E. Courtade, F. Marion, P.J. Nacher, G. Tastevin, T. Dohnalik, K. Kiersnowski, Hyperf. Interact. 127, (2000) 451.

13. M. Abboud, A. Sinatra, X. Mâitre, G. Tastevin, P.-J. Nacher, Europhys. Lett. 68, (2004) 480.

14. M. Abboud, A. Sinatra, G. Tastevin, P.-J. Nacher, X. Maitre, Las. Phys. 15, (2005) 475.

15. A. Nikiel, T. Pałasz, M. Suchanek, M. Abboud, A. Sinatra, Z. Olejniczak, T. Dohnalik, G. Tastevin, P.-J. Nacher, Eur. Phys. J. Spec. Top. 144, (2007) 255.

16. A. Nikiel, G. Collier, B. Głowacz, T. Pałasz, Z. Olejniczak, W. Wegglarz, G. Tastevin, P.-J. Nacher, T. Dohnalik, to be published.

17. W. Heil, J. Dreyer, D. Hoffmann, H. Humblot, E. LelievreBerna, F. Tasset, Physica B 328, (1999) 267.

18. J. Choukeife, X. Mâitre, P. J. Nacher, G. Tastevin, Proc. Intl. Soc. Mag. Reson. Med. 11, (2003) 1391.

19. T. R. Gentile, D. R. Rich, A. K. Thompson, W. M. Snow, G. L. Jones, J. Res. Natl. Inst. Stand. Technol. 106, (2001) 709 .

20. G. Tastevin, S. Grot, E. Courtade, S. Bordais, P.-J. Nacher, Appl. Phys. B 78, (2004) 145.

21. J.H. McLeod, J. Opt. Soc. Am. 44, (1954) 592.

22. B. Depret, P. Verkerk, D. Hennequin, Optics Communications 221, (2002) 31

23. K. Suchanek, M. Suchanek, A. Nikiel, T. Pałasz, M. Abboud, A. Sinatra, P.-J. Nacher, G. Tastevin, Z. Olejniczak, T. Dohnalik, Eur. Phys. J. Spec. Top. 144, (2007) 67.

24. M. Batz, S. Baeßler, W. Heil, E.W. Otten, D. Rudersdorf, J. Schmiedeskamp, Y. Sobolev, M. Wolf, J. Res. Natl. Inst. Stand. Technol. 110, (2005) 293. 